\title{
MARCO LANDOLFI
}

Osservatorio Astrofisico di Arcetri

EGIDIO LANDI DEGL'INNOCENTI

Dipartimento di Astronomia e Scienza dello Spazio, Università di Firenze

\section{MAURIZIO LANDI DEGL'INNOCENTI}

Gruppo Nazionale di Astronomia, Unità di Ricerca di Arcetri

\author{
JEAN-LOUIS LEROY \\ Observatoire Midi-Pyrénées
}

STEFANO BAGNULO

Università di Firenze

\begin{abstract}
Broadband linear polarization in the spectra of Ap stars is believed to be due to differential saturation between $\sigma$ and $\pi$ Zeeman components in spectral lines. This mechanism has been known for a long time to be the main agent of a similar phenomenon observed in sunspots. Since this phenomenon has been carefully calibrated in the solar case, it can be confidently used to deduce the magnetic field of Ap stars.

Given the magnetic configuration of a rotating star, it is possible to deduce the broadband polarization at any phase. Calculations performed for the oblique dipole model show that the resulting polarization diagrams are very sensitive to the values of $i$ (the angle between the rotation axis and the line of sight) and $\beta$ (the angle between the rotation and magnetic axes). The dependence on $i$ and $\beta$ is such that the four-fold ambiguity typical of the circular polarization observations $((i, \beta),(\beta, i),(\pi-i, \pi-\beta),(\pi-\beta, \pi-i))$ can be removed.
\end{abstract}

\section{BLP AND MAGNETIC INTENSIFICATION}

Broadband linear polarization (BLP) observations on Ap stars yield time-varying signals of order $10^{-4}$ (up to $2 \times 10^{-3}$ in the most favourable cases); the variation is rather regular and the period is generally the same as the rotation period of the star deduced from magnetic or photometric measurements (see e.g. Kemp and Wolstencroft 1974).

The same kind of phenomenon (BLP) was previously detected by Dollfus (1958) on sunspots, and studied extensively by Leroy (1962). His observations, 
obtained with a filter having a passband from 4400 to $4900 \AA$, showed a polarization degree of the order of some units times $10^{-3}$, and were interpreted by Leroy himself through a theory based on the so-called magnetic intensification mechanism. This mechanism consists in the different saturation of the $\sigma$ and $\pi$ components of a spectral line splitted by a magnetic field: as a direct consequence of this different saturation, a net linear polarization results after frequency integration over the line profile. According to this mechanism, the polarization observed in a given spectral interval is therefore the cumulative effect of the lines contained in the interval.

Further observations on sunspots were subsequently carried out by Iling et al. (1974a, b), who used a filter with a different passband (from 5250 to $5350 \AA$ ). These observations confirmed the value quoted above for the fractional linear polarization; however, the precise measurement of the direction of linear polarization suggested that magneto-optical effects might play an important role in the physics of radiative transfer. This suggestion was confirmed by the detailed analysis of Calamai et al. (1975), who generalized Leroy's theory and showed that the inclusion of magneto-optical effects resulted in a better agreement between observations and theoretical expectations.

Observations on sunspots support the conclusion that BLP can be successfully explained in terms of the magnetic intensification mechanism, which thus appears to be the natural candidate for the interpretation of the analogous observations on Ap stars. The main observational difference lies in the polarization degree, which is roughly reduced by one order of magnitude compared with sunspots. This difference is probably due to the lack of spatial resolution in stellar observations: since each point of the stellar surface is characterized by its own magnetic field vector, the resulting polarization will be considerably weakened in comparison with the case of a uniform magnetic field.

\section{INTERPRETATION OF BLP IN AD STARS}

According to the magnetic intensification mechanism, the polarization signal from an Ap star depends on the overall number and the characteristics of the spectral lines within the passband, and, most of all, on the geometrical configuration of the magnetic field. The simplest model for the magnetic geometry of the star is to assume a dipolar configuration with the dipole axis tilted with respect to the rotation axis (see Fig. 1), so that a time-dependent polarization results. Under this assumption the BLP can be computed by a frequency integration over the passband and a spatial integration over the stellar disk. Such calculations have been performed by Landi Degl'Innocenti et al. (1981) and have led to a rather satisfactory interpretation of the Kemp and Wolstencroft (1974) observations on the star 53 Cam.

However, the accuracy of the observations does not justify, in most cases, these detailed calculations. For the inversion procedure (i.e. the determination of the magnetic field parameters from polarization observations) a set of analytical - even if approximate - expressions, able to reproduce the basic features of the polarization derived by more rigorous calculations, is certainly more convenient.

Such expressions have been recently obtained by Landolfi et al. (1992) under the weak field assumption $\left(\nu_{L}<\Delta \nu_{D}\right.$, with $\nu_{L}$ the Larmor frequency 


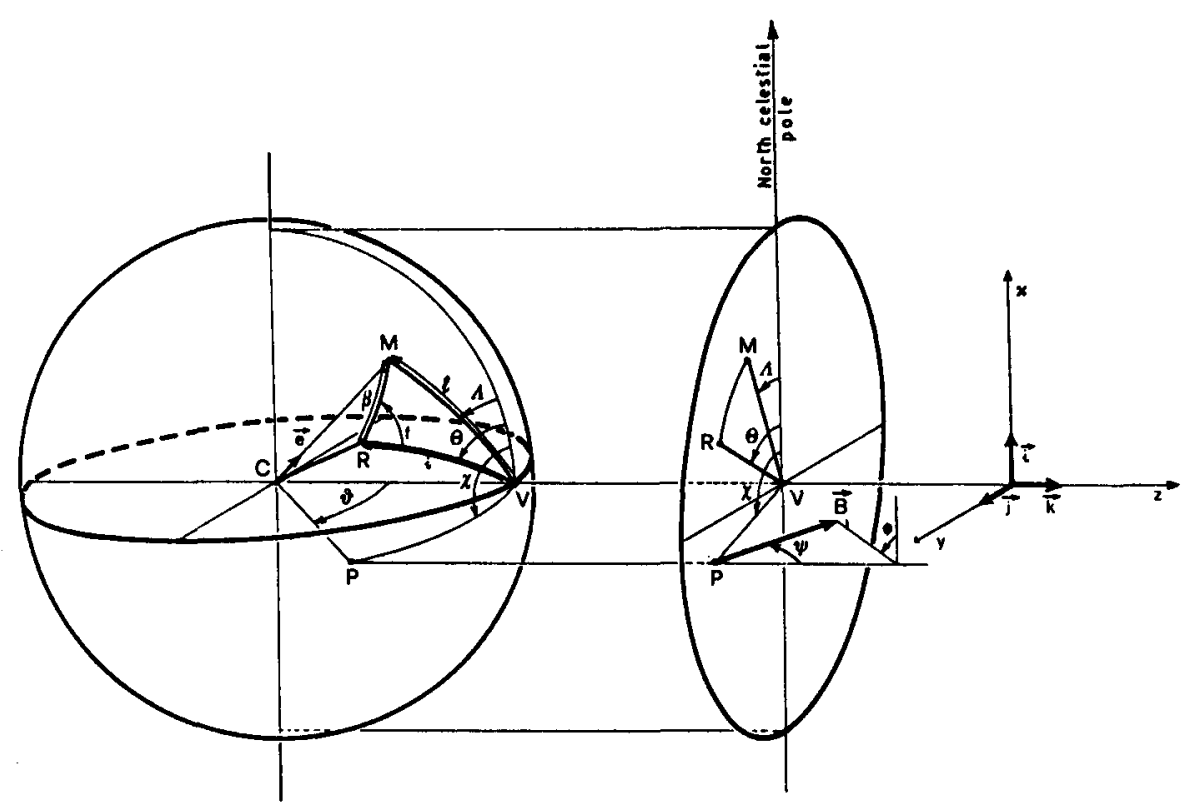

Fig. 1. Geometry of the oblique rotator. CR is the rotation axis, CM is the magnetic dipole axis. The magnetic field vector $\underline{B}$ at any point $P$ (considered fixed on the stellar disk) is specified in the observer's reference frame $(x, y, z)$ by the inclination angle $\psi$ and the azimuth angle $\phi$.

and $\Delta \nu_{D}$ the Doppler width of the line). According to these expressions the Stokes parameters $Q$ and $U$ describing the fractional linear polarization in a given spectral interval can be written as

$$
\begin{aligned}
& Q=k \sin ^{2} l(\cos 2 \Lambda+\epsilon \cos l \sin 2 \Lambda) \\
& U=k \sin ^{2} l(\sin 2 \Lambda-\epsilon \cos l \cos 2 \Lambda)
\end{aligned}
$$

where the time-dependent angles $l$ and $\Lambda$ vary with the rotation period of the star (see Fig. 1), and the quantities $k$ and $\epsilon$ depend on the characteristics of the spectral lines, on the magnitude of the polar magnetic field, and (weakly) on the limb-darkening coefficient. The scale factor $k$ is also roughly proportional to the overall number of spectral lines.

The weak field assumption (commonly used for the interpretation of circular polarization observations) is clearly unrealistic for Ap stars. Nevertheless the simple expressions (1) are able to reproduce faithfully the shape of the polarization diagrams obtained by numerical integrations, provided an appropriate value is chosen for the $\epsilon$ parameter.

The polarization diagrams obtained from Eqs. (1) are very sensitive to the values of the inclination angle $i$ and of the tilt angle $\beta$ specifying the oblique 


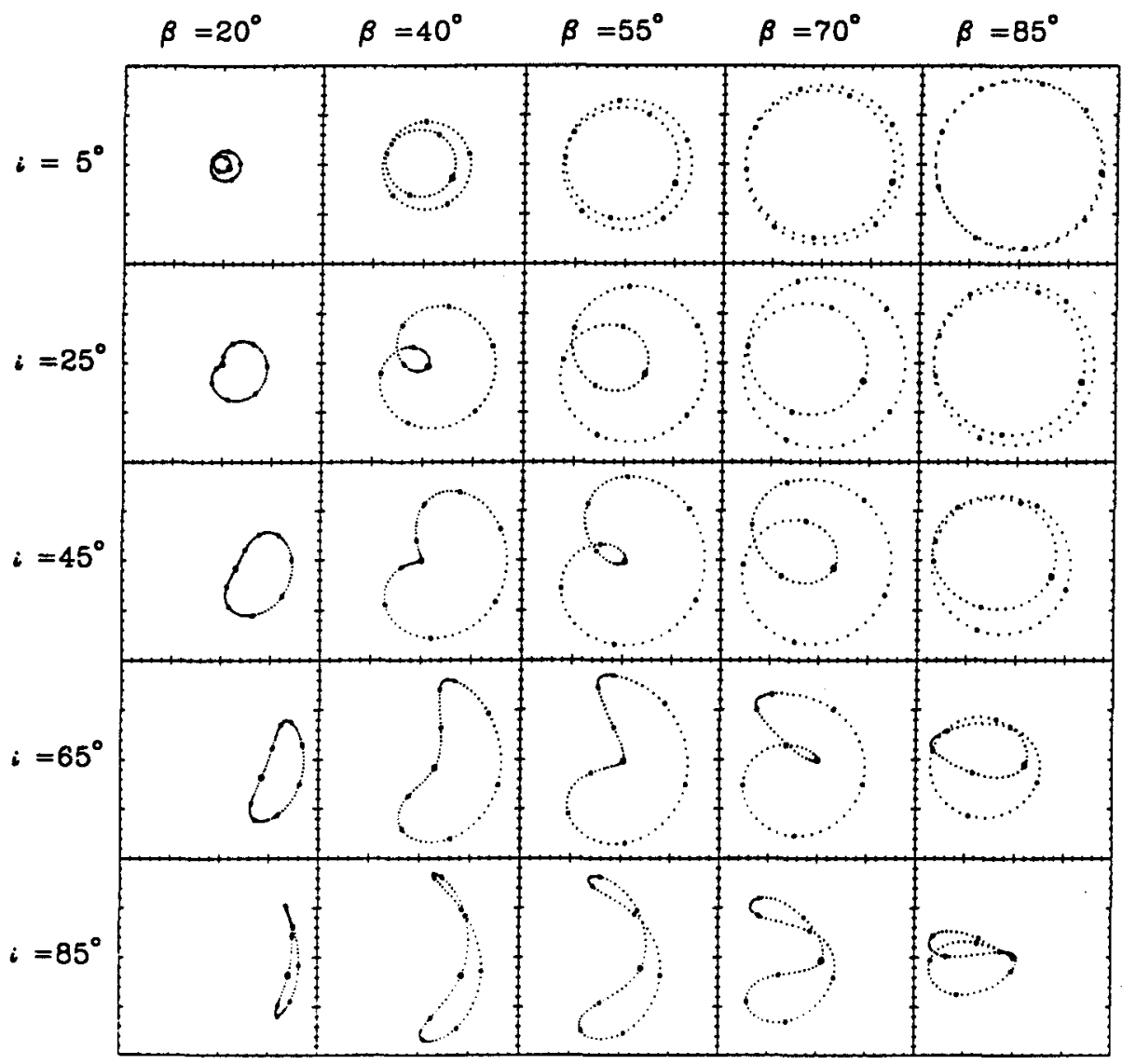

Fig. 2. Polarization diagrams computed from Eqs. (1) (with $\epsilon=0.6$ ) for several values of the $i$ and $\beta$ angles specifying the oblique rotator.

rotator (see Fig. 2). Therefore, even moderately accurate observations should allow to check the reliability of the dipole magnetic field assumption, as well as to determine with some confidence the $i$ and $\beta$ angles. This is an intrinsic advantage of BLP observations compared with the classical circular polarization observations, which do not allow to discriminate between the four configurations $(i, \beta),(\beta, i),(\pi-i, \pi-\beta),(\pi-\beta, \pi-i)$.

New BLP observations on Ap stars have been undertaken by our group with the help of the Pic du Midi 2-meter telescope. An example, concerning the star HD 115708, is shown in Fig. 3. In spite of the observational errors and of the insufficient phase coverage, the appearence of a two-loop diagram can be clearly recognized. The solid line corresponds to $i=127^{\circ}, \beta=97^{\circ}, \Theta=5^{\circ}, f_{0}=276^{\circ}$. 


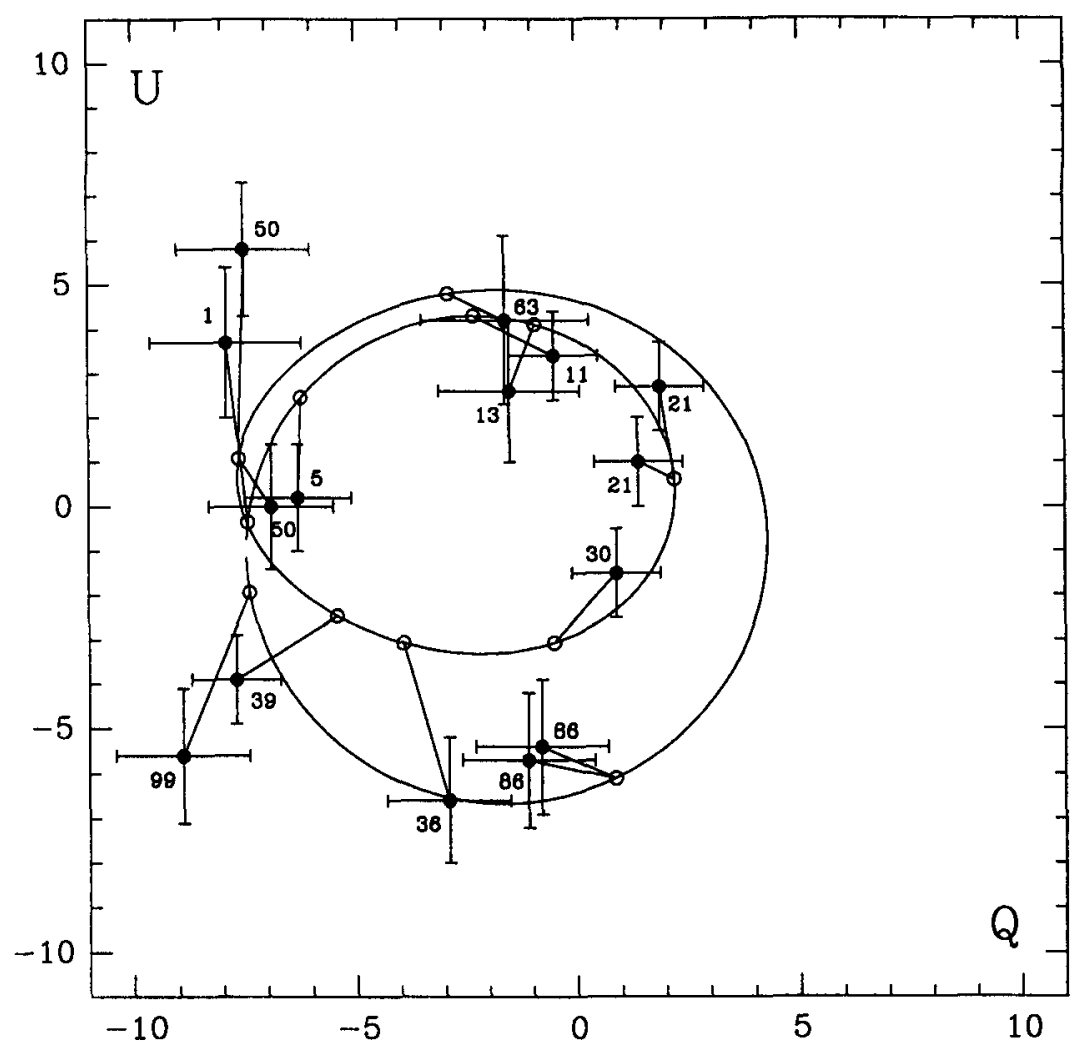

Fig. 3. BLP measurements of the Ap star HD 115708 performed with the Sterenn polarimeter at the Pic du Midi 2-meter telescope. The numbers within the error boxes denote the rotation phase normalized to 100 . The polarization is given in units of $10^{-4}$.

\section{REFERENCES}

Calamai, G., Landi Degl'Innocenti, E. and Landi Degl'Innocenti, M. 1975, Astr. Ap., $\underline{45}, 297$.

Dollfus, A. 1958, Comptes Rendue, 246, 3590 .

Illing, R. M. E., Landman, D. A. and Mickey, D. L. 1974a, Astr. Ap., $\underline{35}, 327$. Illing, R. M. E., Landman, D. A. and Mickey, D. L. 1974b, Astr. Ap., $\underline{37}, 97$.

Kemp, J. C. and Wolstencroft, R. D. 1974, M.N.R.A.S., 166, 1.

Landi Degl'Innocenti, M., Calamai, G., Landi Degl'Innocenti, E. and Patriarchi, P. $1981, A p . J ., 249,228$.

Landolfi, M., Landi Degl'Innocenti, E., Landi Degl'Innocenti, M. and Leroy, J. L. 1992, Astr. Ap., (submitted).

Leroy, J. L. 1962, Annal. Astrophys., 225, 127. 